\title{
Voces de la academia y los tomadores de decisiones del Oriente Colombiano ante la implementación del Modelo Integral de Atención en Salud
}

\section{Voices of the academy and decision-makers from the East of Colombia regarding the implementation of the Integral Health care Model}

\author{
Myriam Ruiz Rodríguez¹, Claudia Milena Hormiga Sánchez², Luz Marina Uribe ${ }^{3}$ Laura del Pilar Cadena Afanador², \\ Blanca Patricia Mantilla Uribe ${ }^{1}$, Sonia Solano Aguilar ${ }^{4}$
}

\begin{abstract}
Forma de citar: Ruiz-Rodríguez M, Hormiga-Sánchez CM, Uribe Rivero LM, Cadena Afanador LP, Mantilla Uribe BP, Solano Aguilar S. Voces de la academia y los tomadores de decisiones del Oriente Colombiano ante la implementación del Modelo Integral de Atención en Salud. Rev Univ Ind Santander Salud. 2017; 49(2): 320-329.

DOI: http://dx.doi.org/10.18273/revsal.v49n2-2017005 (c) (1)
\end{abstract}

\section{RESUMEN}

Introducción: En Colombia, a partir del año 2011 ha resurgido el interés por la incorporación de la Estrategia de Atención Primaria en Salud (APS) en los servicios de salud y en los debates académicos. A partir de la promulgación del nuevo Modelo Integral de Atención en Salud en el año 2016, este interés es explícito por los distintos actores del Sistema General de Seguridad Social en Salud. En el marco de la Alianza por la Atención Primaria en Salud para Colombia, se promovieron encuentros regionales que permitieran identificar potencialidades y limitaciones para la implementación de la estrategia de APS en el marco de la nueva política de atención. Objetivo: Socializar las reflexiones que tomadores de decisiones y representantes de la academia realizaron frente a la implementación de la nueva política de atención en el oriente del país. Metodología: Se realizaron tres mesas de trabajo con 30 actores de 11 Instituciones de Educación Superior y 17 tomadores de decisiones de cuatro departamentos del oriente del país. Se utilizó la estrategia de investigación de grupos de discusión. Resultados: Tanto la academia como los tomadores de decisiones identificaron desafíos relacionados con la comprensión de los aspectos conceptuales del enfoque poblacional del modelo, así como la necesidad de hacer una mirada crítica a las competencias y currículos de los nuevos profesionales. Conclusión: Los distintos temas que emergieron del debate incluyen aspectos estructurales y de funcionamiento del sistema de seguridad social en Salud que le plantean a la autoridad sanitaria retos que superan la mera instrumentalización del modelo.

Palabras clave: Atención Primaria de la Salud; sistemas de salud, gobierno local, reforma de la atención en salud, Colombia.

\footnotetext{
1. Universidad Industrial de Santander. Bucaramanga, Colombia

2. Universidad Autónoma de Bucaramanga, Colombia

3. Secretaría de Salud de Santander, Colombia

4. Universidad de Santander. Bucaramanga, Colombia

Correspondencia: Myriam Ruiz Rodríguez. Dirección: Carrera 32 29-31. Tercer Piso Bucaramanga, Colombia. Correo electrónico: myriam@, uis.edu.co. Teléfono: +76454726 .
} 


\section{ABSTRACT}

Introduction: Since 2011, there has been an increasing interest to re-incorporate primary health care (PHC) strategy in healthcare services and academic debates. After the promulgation of the new healthcare model (MIAS) in 2016, this interest has been made explicit by all actors involved in the health benefits program of the Social Security System (SGSSS). According to the guidelines proposed by the Colombian PHC Alliance, regional meetings have been held to identify potentialities and limitations for implementing the PHC strategy within the framework of the new healthcare policy. Objective: To socialize the analysis carried out by decision-makers and academics related to the implementation of the new healthcare policy in the east of the country. Methodology: Three workshops were held with the participation of 30 representatives of 11 higher-education institutions and 17 decision makers from four departments of the east of the country. Qualitative research methodology based on focus groups was applied. Results: Academics and decision-makers identified challenges related to understanding the conceptual aspects of the Model's population focus and raised awareness about the need of a critical assessment of curricula and professional competencies of health professionals. Conclusion: The discussion topics emerged from the debate included structural and functional aspects of the Health System that represent challenges to health authorities beyond the mere operationalization of the model.

Keywords: Primary health care, Health Systems, local government, Health care reform

\section{INTRODUCCIÓN}

Aunque existe suficiente evidencia de que los países que incorporan en sus sistemas de salud la estrategia de Atención Primaria en Salud (APS) tienen mejores resultados en salud ${ }^{1,2}$, en Colombia esta estrategia desapareció del contexto de los servicios de salud con la implementación de la Ley 100 de 1993².

No obstante, en los últimos años, ha resurgido en el país el interés por incorporar la APS en los modelos de atención y son varias las experiencias que han sido socializadas y evaluadas en el contexto del Sistema General de Seguridad Social en Colombia (SGSSS) ${ }^{4,5}$. En ellas se identifican problemas como la falta de voluntad política, la inexistencia de normatividad para incorporar dicha estrategia a los servicios, la intermediación de los servicios, la fragmentación y el desconocimiento mismo de la APS como una estrategia básica para lograr mejoras en la salud.

Pero no fue sino hasta después de la promulgación de la Ley 1438 de $2011^{6}$ y del Plan Decenal de Salud Pública (PDSP) 7 en el año 2012 que reemergió, en los actores institucionales del sector de la salud y de la educación, la necesidad de mejorar el conocimiento de la APS, así como de identificar estrategias que faciliten su incorporación en los modelos de atención y de formación de talento humano en salud.

En la Ley 1438 se establece cómo las acciones de promoción y prevención en el ámbito de la salud pública y en el de las actividades individuales de baja complejidad pueden desarrollarse y financiarse en el contexto de la estrategia de APS. Sin embargo, como lo evidencian Vega, et al. "en la práctica, estos recursos fueron usados discrecionalmente por cada uno de los actores del SGSSS en la actividad respectiva de su competencia, con escasa o sin ninguna concurrencia real de las diversas fuentes en el financiamiento de las actividades de los equipos básicos de salud de modo que se permita la atención integral que deberían prestar bajo la estrategia de APS". Otros trabajos han mostrado las distintas limitaciones y retos que los sistemas de salud han venido afrontando para la implementación de la APS 9 .

En este contexto, en el año 2014 surgió la iniciativa Alianza por la APS para Colombia, con el propósito de fortalecer la construcción, implementación, seguimiento y evaluación de un modelo de atención basado en $\mathrm{APS}^{10}$. Esta iniciativa, liderada por el Instituto Nacional de Salud, estuvo respaldada por la Organización Panamericana de la Salud en Colombia, el Ministerio de Salud y Protección Social, el Instituto Nacional de Salud, la Facultad Nacional de Salud Pública de la Universidad de Antioquia, la Dirección de postgrados en Administración en Salud de la Pontificia Universidad Javeriana de Bogotá y la Corporación de Secretarios de Salud Municipal - COSESAM. Las acciones realizadas en el marco de esta iniciativa tomaron mayor importancia con la promulgación de la Resolución 429 de 2016, que dicta la nueva Política de Atención Integral de Salud (PAIS) ${ }^{11}$.

Esta política cuenta con un marco estratégico fundamentado en cuatro estrategias centrales: la APS con enfoque familiar y comunitario, la gestión integral del riesgo en salud, el cuidado y el enfoque diferencial. De igual manera propone un modelo operacional, 
Modelo Integral de Atención en Salud (MIAS), que a partir de las cuatro estrategias adopta herramientas para garantizar la oportunidad, continuidad, integralidad, aceptabilidad y calidad de la atención en salud de la población bajo condiciones de equidad ${ }^{11}$.

La Alianza por la APS para Colombia buscó consolidar una red nacional de investigación e implementación en APS, para lo cual planteó cuatro líneas estratégicas: a) generación, diseminación y uso de conocimiento; b) apoyo al desarrollo de capacidades y entrenamiento para la APS; c) construcción de redes de apoyo; y d) incidencia en las políticas sobre APS. Así mismo definió para el año 2015, realizar encuentros regionales (regiones Atlántico, Pacífico, Orinoquía, Centro y Oriente) que propiciaran el reconocimiento de experiencias exitosas desde el ámbito de los servicios de salud y de la investigación en APS, la generación de espacios de diálogo y la identificación de necesidades para priorizar agendas de investigación en APS. Estos encuentros fueron coordinados y liderados por el director de la Subdirección de Innovación de la Dirección de Investigación en Salud Pública, del Instituto Nacional de Salud.

En cabeza de la Secretaría de Salud de Santander (SSS) y del Departamento de Salud Pública de la Escuela de Medicina de la Universidad Industrial de Santander (UIS) quedó la responsabilidad de propiciar el diálogo entre actores de la academia y del sector salud para la región oriental del país (departamentos de Cesar, Arauca, Norte de Santander y Santander), para dar origen al Nodo Oriental de la Alianza. Con base en lo anterior, hasta el momento se han realizado dos encuentros del nodo..

El segundo encuentro se celebró en abril de 2016 y contó con participantes de la academia, de las Secretarías Departamentales de Salud y de algunas Direcciones Locales de Salud, asesores del Viceministerio de Salud Pública y Prestación de Servicios del Ministerio de Salud y Protección Social (MSPS). El tema de este encuentro fue "Atención Primaria en Salud en el marco del Modelo Integral de Atención en SaludMIAS" y su propósito fue socializar la nueva política PAIS y hacer un ejercicio de reflexión, por parte de la academia y de los entes territoriales en salud, para reconocer fortalezas, debilidades y oportunidades para la implementación de la nueva política en los territorios de la región.

En este artículo se presentan los resultados de las mesas de trabajo realizadas en el contexto de este segundo encuentro. Cabe resaltar que una mirada crítica de la
PAIS y su modelo operativo, su pertinencia o viabilidad en el contexto actual del país, va más allá del alcance de este artículo.

\section{METODOLOGÍA}

Previo al encuentro se realizó un proceso metodológico de dos fases. Para la primera, la SSS y la UIS convocaron al equipo base de la alianza en el departamento de Santander, conformado por funcionarios de la SSS y representantes de universidades con experiencia en la gestión de salud pública y conocimiento del nuevo modelo de atención en salud. Este grupo realizó un primer análisis sobre las dificultades que los servicios de salud a nivel local y departamental han reportado para la implementación de la estrategia de APS, en el entendido, que la mayoría de estas experiencias se desarrollaron antes y recién promulgada la Ley 1438 y el PDSP. Para ello se tomó como base para el análisis, las experiencias de APS presentadas en el primer encuentro de la Alianza por la APS del Nodo Oriente. A modo de conclusión de esta primera fase, se identificó la heterogeneidad de las experiencias y en especial, el papel que los distintos actores desempeñaron en dichas experiencias y la capacidad de respuesta de las autoridades y prestadores de salud para movilizar recursos intersectoriales.

Una segunda fase, consistió en el análisis y discusión del marco estratégico de la política PAIS, haciendo énfasis en la estrategia de APS, y los componentes operacionales del MIAS. En este sentido, se definió una matriz de análisis, en la que se identificaron los distintos actores implicados en la implementación del MIAS, algunos retos que se deben superar para realizar la transformación del actual modelo de atención, así como las implicaciones para los tomadores de decisiones, en cuanto a necesidades y apoyos requeridos para realizar esta transformación.

Como resultado del ejercicio de análisis, la SSS y el Departamento de Salud Pública de la UIS definieron la metodología de las mesas. Se convocaron a Secretarios de Salud o sus delegados de las capitales de los cuatro departamentos y sus municipios y a miembros de la academia de las Facultades de Salud (directivos y profesores) de todas las universidades que tuvieran presencia en estos departamentos $\mathrm{y}$ que entre sus planes curriculares incorporaran contenidos y prácticas de APS mediante convenio docencia asistencia con los servicios de salud. Cada institución y ente territorial financió la asistencia de los participantes. 
Se desarrollaron tres mesas de trabajo, dos con representantes de la academia y una con tomadores de decisión en los ámbitos departamental y municipal. Estas mesas siguieron una metodología de grupos de discusión ${ }^{12}$ y el equipo de análisis elaboró las preguntas disparadoras para tal efecto. Cada mesa contó con un moderador, un relator y un observador.

Las mesas de trabajo de la academia estuvieron conformadas por 30 participantes procedentes de 11 universidades (tres públicas y ocho privadas); 36,3\% de las universidades tiene presencia en tres departamentos, $8 \%$ en dos departamentos y el 55,7\% en el departamento de Santander. Los objetivos que guiaron el debate fueron: a) identificar necesidades de formación del talento humano en la región que facilite la implementación del Modelo Integral de Atención en Salud y b) explorar el apoyo que las instituciones educativas pueden proporcionar a los entes territoriales y las Instituciones de Salud para la implementación del MIAS.

En la mesa de tomadores de decisión participaron 17 personas que desempeñaban cargos de alto nivel directivo en las Secretarías de Salud Departamental y Municipal, así como de Institutos descentralizados en salud procedentes de las capitales de los cuatro departamentos y de otros 13 municipios del Nodo Oriente. Un representante del Viceministerio de Salud Pública y Prestación de Servicios del MSPS hizo parte de esta Mesa de Trabajo. Dos objetivos guiaron la discusión: a) Identificar fortalezas y debilidades en los territorios para la implementación del MIAS, con el enfoque de APS y b) determinar los apoyos que requieren los entes territoriales del nivel local, departamental para la implementación del MIAS.

Debido a las características de la convocatoria y a las experiencias de los participantes las discusiones se centraron en las necesidades, debilidades y retos para la implementación del MIAS en lo referente a la estrategia de APS, que como ya se anotó es una de las cuatro estrategias centrales de la PAIS. En este sentido, el concepto de APS que se tuvo en cuenta para las discusiones fue el definido para la nueva política que considera a la APS como una estrategia integradora entre las necesidades de la población, la respuesta del sistema y los recursos disponibles en la sociedad como elemento esencial para la viabilidad en la progresividad del ejercicio del derecho. Una APS con enfoque de salud familiar y comunitaria que sirve de interfaz entre la necesidad de desarrollo de nuevas competencias del recurso humano y equipos interdisciplinarios empoderados en el desarrollo de sus capacidades para dar una respuesta social dirigida hacia la persona, la familia y la comunidad ${ }^{11}$.

Las sesiones de cada mesa fueron grabadas en audio, previa solicitud a sus participantes. Los audios fueron transcritos y siguiendo el análisis de contenido ${ }^{13}$ se identificaron temas relevantes de la discusión que emergieron de cada una de las preguntas disparadoras y posteriormente fueron agrupados en los temas que se presentan en el presente artículo.

\section{RESULTADOS}

En general, los participantes del encuentro reconocieron la importancia de aprovechar el momento coyuntural que experimenta el país ante la promulgación de PAIS, celebran que la APS sea una de las estrategias centrales de esta política y manifiestan su interés de participar ampliamente en su implementación, pues son conscientes de la necesidad de mejorar el sistema sanitario del país. No obstante, expresan su preocupación por el verdadero alcance de la implementación del MIAS, dado que esta normativa propende por una reorientación de la atención sanitaria y el fortalecimiento de la salud pública, pero no representa un cambio estructural en el sistema de salud.

\section{Temáticas identificadas por la academia}

\section{Necesidades de formación del talento humano para la implementación del MIAS en la región}

- Fortalecimiento del enfoque comunitario y de Atención Primaria en Salud en los programas del área de la salud

En su mayoría, los actuales programas académicos del área de salud que se ofertan en la región incluyen contenidos de los enfoques comunitario y de determinantes sociales de la salud, pero adolecen de un claro enfoque en APS. Adicionalmente sus estructuras curriculares son fuertemente disciplinares por lo tanto no fomentan el trabajo interdisciplinario e intersectorial. Por esta razón se hace imprescindible una revisión profunda en los currículos con el objetivo de fortalecer estos enfoques, teniendo presentes el contexto social, económico y político local, para permitir a los futuros profesionales apropiarse de un principio y es que la salud es resultado personal pero y sobretodo social, en el cual el trabajo de equipos interdisciplinarios e intersectoriales son fundamentales. 
- Directrices sobre el desempeño de los perfiles ocupacionales que se integran en la estrategia de APS.

Los roles de los diferentes perfiles laborales del área de la salud poblacional no han sido definidos por el Ministerio de Salud y Protección Social para la implementación de la estrategia de APS en el MIAS. Por esta razón se plantea la necesidad de que el Ministerio de Salud establezca lineamientos sobre el desempeño esperado de cada perfil en el modelo de atención, partiendo del entendimiento de que todos los perfiles son requeridos en la implementación y liderazgo del modelo, y que de la integración y complementariedad de los miembros de los equipos interdisciplinarios depende la provisión de una atención integral en salud. Estos roles deben corresponderse con unas competencias de formación transversales enfocadas en la APS y otras competencias específicas para cada perfil profesional, las cuales deben ser fortalecidas en los currículos de los programas del área de la salud.

Al respecto se planteó la necesidad de acompañar la iniciativa conjunta de los Ministerios de Salud y la Protección Social y el de Educación con relación al establecimiento de unas competencias específicas para cada perfil laboral, con el fin de contar con unos lineamientos acordes a las necesidades de las regiones y del país. Así mismo, de identificar competencias transversales a todos los perfiles con fuerte énfasis en APS.

De manera específica, los participantes presentaron la necesidad de fortalecer la participación y formación del profesional en psicología en el área de la salud, haciendo énfasis en la promoción de la salud y prevención de la enfermedad y no en la atención clínica y rehabilitación.

- Formación en Medicina Familiar, Salud Familiar y otros perfiles requeridos en el nuevo modelo de atención

Se plantea la urgencia de formación de profesionales de salud especializados en Medicina familiar y Salud familiar que lideren el proceso en las regiones. En la actualidad hay un escaso número de profesionales de la salud con esta formación en la región, la mayoría de los cuales no se están desempeñando como profesionales especializados, dado que hasta ahora su figura no ha sido relevante en el modelo de atención sanitaria del país, ni se han dado espacios para que desarrollen su campo de acción.

Asimismo, se resalta la necesidad de formación de los nuevos perfiles, por ejemplo el de tecnólogo en promoción de la salud. En este sentido se hace necesario crear programas de formación en las instituciones de educación superior, en cuyos currículos se incorpore la APS y se establezcan las competencias que este perfil de formación requiere para rescatar el contacto cercano del personal de salud con la comunidad y fortalecer la puerta de entrada al sistema sanitario, así como la identificación de las necesidades de salud de la población.

- Creación de una cátedra dentro de cada universidad dirigida a promover el trabajo intersectorial en salud, en la que participen diversas áreas del conocimiento, como los programas de ingenierías, social-humanísticas, entre otras.

- Incluir la formación en sistemas de información para la toma de decisiones $y$, en este sentido, promover la calidad de los registros poblacionales y la cultura de la evaluación centrada en los resultados de salud. Específicamente en los programas de posgrados de salud poblacional es fundamental que se promuevan las competencias en el diseño, evaluación y administración de los sistemas de información aplicados en salud.

2. Estrategias de apoyo de las instituciones de educación superior (IES) a los entes territoriales para la implementación del MIAS

Las universidades aportan a la sociedad desde sus tres pilares misionales que son docencia, investigación y extensión. En cada uno de estos se propusieron estrategias que favorecen la implementación del MIAS en la región.

\section{- Docencia}

Se identifica que desde la docencia las IES deben apoyar dos grupos prioritarios: los futuros profesionales y los profesionales que ya se encuentran en ejercicio, con el fin de que incorporen la estrategia de APS en su labor diaria, pues son quienes realizarán la implementación del modelo.

Con relación al primer grupo se planteó la necesidad de realizar alianzas entre las IES, en procura de la solución de las necesidades de formación de talento humano identificadas en la región. Esto implica hacer acuerdos institucionales con metas programáticas de formación, identificando las fortalezas de cada universidad y, en un espíritu colaborativo, diseñar estrategias de financiamiento e incentivos, tanto para los futuros profesionales, como para las IES. 
Con relación al grupo de profesionales en ejercicio, se planteó la necesidad de realizar, bajo el marco de convenios, capacitación y educación continua en la estrategia de APS, y el reconocimiento de los determinantes sociales que intervienen en las condiciones de salud de las poblaciones.

\section{- Investigación}

Se reconocen las fortalezas que las universidades de la región tienen en investigación sobre APS y el estado de salud de las comunidades, las cuales se constituyen en la línea de base para el inicio de la implementación del MIAS. Por lo tanto se propusieron los siguientes aspectos, como elementos clave a desarrollar en este pilar misional:

- Acompañara los entes territoriales en el seguimiento, monitoreo y evaluación de la implementación del MIAS en tres ámbitos: sistemas de información, gestión del modelo y resultados en salud.

- Apoyar a los entes territoriales en la resolución de sus necesidades de investigación y gestión sanitaria, a partir de las cuales se puede reestructurar parte del trabajo de las instituciones educativas.

- Estudiar los incentivos que tienen los profesionales de salud para desempeñarse en el nuevo modelo de atención. Se propone realizar estudios de equivalencias administrativas y financieras entre las atenciones clínicas usuales y las atenciones enmarcadas en el enfoque de medicina familiar y APS, con el fin de promover la atención bajo este último enfoque.

- Generar proyectos colaborativos interdisciplinarios que incluyan formulación y generación de propuestas innovadoras que incluyan la evaluación de sus resultados e impacto.

- Fortalecer la implementación del enfoque de investigación-acción-participación en investigaciones consensuadas y financiadas por los entes territoriales. De esta manera generar evidencias de efectividad que fortalezcan la toma de decisiones locales con base en el contexto y los actores allí presentes.

- Indagar a profundidad las percepciones que los profesores y los estudiantes tienen sobre el enfoque comunitario y la APS, con el fin de identificar áreas críticas que contribuyan a la formación integral del profesional del área de la salud. De otro lado, es conocido que en las universidades el eje curricular orientado a la salud pública tiene una falta de valoración desde el currículo oculto, este aspecto amerita ser estudiado para proponer estrategias de intervención.
- Visibilizar el efecto que la falta de estabilidad laboral y la alta rotación de los profesionales de salud tienen en el desempeño y sostenibilidad de las instituciones, proyectos, programas y en la salud de las poblaciones.

\section{- Extensión}

Es importante rescatar la invitación que los diferentes entes territoriales hacen a las universidades de la región para que apoyen la implementación del nuevo modelo de atención. En correspondencia, los representantes de las universidades proponen emplear el pilar de la articulación universidad con la sociedad para proveer evidencias sobre experiencias exitosas que orienten la implementación del modelo, procurando que estas trasciendan los cambios de gobierno. Para esto se propone la incorporación de procesos de la academia en la implementación de la APS y el MIAS que lideran los entes territoriales, teniendo presente que la responsabilidad de esta implementación recae sobre éstos y no en las universidades.

Así mismo, se propone identificar las capacidades instaladas y fortalezas de las universidades para articularlas a las necesidades y expectativas de los diferentes actores del sistema sanitario con el propósito de apoyar la puesta en marcha del modelo de atención.

\section{Temáticas identificadas por los tomadores de decisiones}

Los participantes de las secretarías de salud departamentales y municipales manifestaron su preocupación por la falta de capacidad institucional y organizativa para implementar el MIAS. Específicamente, perciben al modelo muy complejo y no tienen claridad conceptual, ni procedimental para la aplicación. Así mismo, se reconoce que la implementación requerirá de un fuerte compromiso de los distintos actores del SGSSS (en especial el ejercicio de rectoría del MSPS) y la voluntad política de los gobernadores y alcaldes para liderar la movilización de los distintos actores y recursos del territorio que garanticen el trabajo inter y trans-sectorial necesario para lograr la implementación del modelo con el enfoque de derechos y de determinantes sociales de la salud

\section{Fortalezas y debilidades}

En general, esta mesa discutió en mayor proporción las debilidades y retos, más que las fortalezas existentes para dar respuesta al nuevo modelo. Las fortalezas 
fueron identificadas por los tomadores de decisiones de los departamentos de Santander y Arauca. Santander en lo referente a la alianza existente entre la Secretaría de Santander y la Universidad Industrial de Santander (UIS).

Esta alianza ha permitido el desarrollo de un trabajo de varios años orientado al fortalecimiento y desarrollo de las capacidades del talento humano en APS y, en el último año éste se ha orientado al desarrollo de competencias de los municipios para la adopción e implementación del MIAS. Igualmente, se socializó, cómo el departamento de Arauca y Santander incluyeron en sus planes de desarrollo, actividades y estrategias que favorecerían la implementación del modelo. Al respecto se hace notar la importancia que los nuevos gobiernos tienen en la aplicación de la nueva normatividad. La voluntad política para incorporar en las agendas públicas, estrategias, acciones y recursos será fundamental para la implementación del modelo.

El debate sobre las debilidades se centró en las siguientes temáticas:

- Concepciones sobre la salud y los servicios: preeminencia del enfoque económico en el sector, mirada patocéntrica de la salud y enfoque curativo de los servicios de salud. De igual manera, hay un mayor desarrollo de los servicios de mayor complejidad y niveles de atención dos y tres, mientras que en los del primer nivel hay poca capacidad resolutiva.

- Escaso número de profesionales trabajando en lo local con enfoque de salud pública; esto se debe a que la mayoría de los profesionales que está en los municipios está cumpliendo con el servicio social obligatorio y por lo tanto su capacidad resolutiva es baja; adicionalmente, se indica que en el departamento de Santander existe dificultad para vincular personal de salud para desempeñarse en áreas rurales y pequeños municipios.

- Inexistencia/deficiencia de los sistemas de información a nivel institucional y del territorio. La mayoría de los territorios del nodo no cuenta con un sistema de información institucional y comunitario que facilite la toma de decisiones de los distintos actores que confluyen en lo local. El grupo hace notar que disponer de un sistema que integre información, del sector salud y de otros sectores, permitirá desarrollar los elementos y procesos de la gestión integral del riesgo en salud.

- Apatía de instituciones y funcionarios de la salud en lo local asociada a: a) la alta rotación del personal de salud, b) la ausencia de consensos, c) la limitación de recursos, d) dificultades en la relación con las empresas administradoras y las prestadoras locales y e) la falta de voluntad política para articular acciones que afecta el trabajo intersectorial e interinstitucional.

- Débil coordinación intrasectorial en lo local y regional. Se identifican dificultades en la integración de actores al interior del propio sector salud: Aseguradoras, Municipios, Prestadoras de servicios de salud, que dificulta la acción de los entes territoriales como autoridad sanitaria. Además, el grupo señala, cómo esta falta de coordinación se ve reflejada en fallas del sistema de referencia y contra referencia, pues son las personas que viven en municipios pequeños y la población rural dispersa la que tiene mayores dificultades para tener un real acceso a los servicios de salud y un alto gasto de bolsillo. A lo anterior se suman las grandes barreras geográficas y culturales para el acceso efectivo a los servicios de salud.

\section{Apoyos requeridos para la implementación del MIAS}

Se identificaron tres actores clave para la implementación del MIAS en los municipios. El primer actor fue el MSPS, catalogado como el actor que debe liderar el cambio con una amplia mirada a la diversidad de los municipios, especialmente a los más pequeños y con población rural dispersa. Los integrantes de la mesa confían en que el MSPS ejerza también una adecuada rectoría del sistema con el fin de garantizar un desarrollo armónico del modelo.

El segundo actor es la autoridad de salud departamental, quien debe hacer el acompañamiento del proceso y dar celeridad en la concurrencia de recursos con los distintos municipios; consideran los participantes que las Secretarías de Salud Departamentales deben centrarse en definir estrategias que permitan vislumbrar cómo se van a organizar las rutas y redes acorde a las realidades geográficas, orográficas, poblacionales y de disponibilidad de instituciones de salud; es decir, se requiere un gran apoyo del nivel departamental para que se definan rutas pensando en la gente y no sólo en la rentabilidad económica.

De otro lado, se discutió el rol preponderante que tendrá el nivel departamental en lo referente a la movilización de recursos y actores departamentales y municipales que garanticen el enfoque inter $\mathrm{y}$ transectorial que requiere el modelo. Este apoyo debe traducirse en la incorporación en los planes de desarrollo y planes 
operativos de acciones que pongan en la agenda pública la implementación del modelo.

El tercer actor es la academia. En este sentido, hacen énfasis en que las instituciones educativas de nivel técnico y superior pueden apoyar la implementación del modelo innovando en las formas de llevar capacitación continua a los profesionales que ya trabajan en los municipios y realicen reformas curriculares para fortalecer la capacidad de los futuros profesionales para hacer intervenciones con enfoque comunitario y familiar.

\section{Retos}

En general, los participantes discutieron diferentes desafíos. Tres fueron los más significativos: la apropiación del modelo, el desarrollo de una cultura de atención basada en la salud como un proceso social, y el fortalecimiento de capacidades y competencias institucionales y organizacionales que den sustento al modelo de atención. Estos desafíos atañen prácticamente a todos los actores del sistema de salud, no obstante, el último reto implica directa y exclusivamente a las autoridades nacionales y departamentales de Salud. Tanto el MSPS, como las autoridades departamentales, deben crear escenarios, estancias y herramientas que permitan la apropiación del modelo en lo conceptual $\mathrm{y}$ en lo instrumental $\mathrm{u}$ operativo que faciliten la comprensión del enfoque de determinantes sociales y su operacionalización en el territorio, es decir, se debe orientar en el cómo implementar el modelo.

\section{DISCUSIÓN}

Los hallazgos de las mesas de trabajo conformadas por miembros del sector académicos y del nivel directivo de los entes territoriales evidencian debilidades, retos y desafíos para la implementación del MIAS en la región Oriental de Colombia.

Desde hace varios años en Colombia ${ }^{8,14}$, así como en América del Sur ${ }^{9}$ se viene documentando desde distintas perspectivas las dificultades que existen para la implementación de la estrategia de APS. El presente trabajo reportó temáticas que también han sido señalados en dichas investigaciones.

Un eje crítico identificado en este estudio, tanto por los participantes de la academia, como los del nivel directivo en salud fue el del talento humano. En tanto que la academia identifica como una prioridad la necesidad de contar con nuevos perfiles, el ajuste de currículos para mejorar las competencias de salud pública de los egresados, y la necesidad de definir normatividad para el desempeño laboral de los profesionales en APS, los tomadores de decisiones reclaman de la academia su concurso para que los profesionales ya vinculados al SGSSS se apropien, conceptual, metodológica y procedimentalmente de la nueva política de atención que supere el enfoque centrado en la enfermedad, el escaso número de profesionales trabajando que tienen el enfoque de salud pública y la alta rotación derivada de la falta de estabilidad laboral.

La necesidad de contar con talento humano calificado para trabajar en contextos de APS ya ha sido reportado por otros estudios ${ }^{14}$ y es conocido que países como Chile, que ha logrado importantes avances en APS, incorporó un estatuto de contratación de personal en el sistema de atención primaria. Dada la importancia que la gestión del talento humano tiene en las instituciones ${ }^{15}$, este tema debe ser central para la implementación del MIAS.

Acorde con los resultados de esta investigación, aspectos como la definición de competencias de APS de los profesionales actuales, el desarrollo de capacidades para el enfoque comunitario y familiar y la necesidad de nuevos perfiles son retos para los tomadores de decisiones del ámbito nacional y departamental. Mientras no se resuelvan estos aspectos en la política de talento humano seguirán trabajando profesionales de salud que no están formados apropiadamente para responder de manera adecuada a las exigencias del MIAS.

Diversos países han definido perfiles de competencias para los equipos de APS centrados en el papel de las y los profesionales en medicina y enfermería. En Colombia, como lo notaron Rey-Gamero y Acosta-Ramírez ${ }^{16}$, se ha definido una guía metodológica para la definición de competencias laborales que requieren adaptación para la implementación de APS. La definición de estas competencias es un aspecto relevante para las IES, pues la implementación del MIAS requiere que los programas de pregrado y posgrado estén en sintonía con el nuevo modelo de atención y, por tanto, con la oferta laboral para sus egresados.

En el presente trabajo se destaca como la alta rotación del talento humano, probablemente favorecida por políticas de flexibilización laboral con contratos de corta duración y discontinuos, y el escaso reconocimiento o falta de incentivos para trabajar en regiones rurales y áreas lejanas, sería un obstáculo para desarrollar el 
componente territorial del MIAS. Al respecto cabe mencionar que si bien la legislación colombiana ha reconocido el rol fundamental del talento humano en el desarrollo de las estrategias en salud, las condiciones laborales que se ofrecen en las instituciones de salud al parecer no dan cuenta de lo que la legislación establece ${ }^{17}$.

Los participantes de este estudio también reportaron barreras para la implementación del MIAS relacionadas con la estructura del SGSSS siendo las más frecuentemente mencionadas, la falta de capacidad institucional y organizativa para implementar el MIAS, el énfasis económico del sector salud, las fallas en la coordinación entre actores y la falta de rectoría. Estas barreras fueron señaladas principalmente por la mesa de tomadores de decisiones y coinciden con lo que otros estudios han reportado, en donde se señala que la estructura organizativa y programática del SGSSS no facilita el desarrollo de estrategias integradoras como lo es la APS $^{6,14}$ no favorece el acceso ${ }^{18}$ y priman los intereses de lucro en las decisiones clínicas y administrativas $^{19}$.

Otro resultado que fue destacado, tanto por la academia, como por los tomadores de decisiones fueron las potencialidades que representa el establecimiento de alianzas entre los entes territoriales y las IES. Se visualiza por parte de todos los integrantes de las mesas como estas alianzas pueden generar proyectos de investigación y extensión para promover las buenas prácticas en el ámbito de la intervención interdisciplinaria y transdiciplinaria, la implementación del enfoque de investigación acción y sobre todo las sinergias para desarrollar investigaciones sobre la implementación del MIAS.

La investigación sobre la implementación de políticas ayuda a aclarar cómo el contexto favorece o no el desarrollo de éstas y sus resultados ayudan al seguimiento en la implementación de las mismas. Es una herramienta útil para ayudar a las organizaciones a fortalecer su capacidad de aprendizaje con miras a asimilar y poner en práctica los conocimientos desarrollados regularmente ${ }^{20}$. Las alianzas inter institucionales e intersectoriales son críticas para Colombia en estos primeros años de la implementación del modelo porque la unión de intereses permite hacer un uso eficiente de los recursos disponibles y enfocarlos a las necesidades prioritarias ${ }^{21}$.

Finalmente, el presente estudio permitió escuchar de primera mano los retos que los tomadores de decisiones y representantes de la academia del oriente colombiano tendrán que enfrentar para la implementación del MIAS en el marco de la estrategia de APS. Los temas discutidos mayormente se relacionaron con la formación del talento humano, las barreras del SGSSS en términos de su estructura y la conformación de alianzas entre las IES y los entes territoriales. El MSPS, las entidades territoriales de salud y el sector académico deberían considerar esta información con el fin de diseñar estrategias que faciliten el desarrollo del MIAS en una forma articulada.

\section{AGRADECIMIENTOS}

A la Universidad Industrial de Santander y la Gobernación de Santander - Secretaría de Salud Departamental por la financiación del presente trabajo, producto del contrato interadministrativo No. 3947 de 2015; a las profesionales Celmira Vesga Gómez y Leonor Jaimes García por su apoyo en la logística y organización y a los integrantes de las mesas de trabajo.

\section{REFERENCIAS}

1. Labonté R, Sanders D, Baum F, Schaay N, Packer C, Laplante D, et al. Aplicación, efectividad y contexto político de la atención primaria integral de salud: resultados preliminares de una revisión de la literatura mundial. Rev Gerenc Polit Salud. 2009; 8(16): 14-29.

2. Starfield B, Shi L, Macinko J. Contribution of primary care to health systems and health. Milbank Quarter. 2005; 83(3): 457-502. DOI: 10.1111/j.14680009.2005.00409.x.

3. Congreso de la República de Colombia. Ley 100 de 1993. Diario Oficial No. 41.148 (23-12-1993).

4. Rodríguez-Villamizar LA, Ruiz-Rodríguez M, Acosta-Ramírez N. Evaluación de un modelo de atención primaria en salud en Santander, Colombia. Rev Fac Nac Salud Pública. 2016; 34(1): 8895. DOI: http://dx.doi.org/10.17533/udea.rfnsp. v34n1a11.

5. Mosquera PA, Hernández J, Vega R, Junca C. Experiencia de implementación de la estrategia de atención primaria en salud en la localidad de BOSA. Rev Gerenc Pol Salud. 2011; 10(21): 124-152.

6. Congreso de la República de Colombia. Ley 1438 de 2011. Diario Oficial No. 47.957 (19-01-2011).

7. Ministerio de Salud y Protección Social. Plan decenal de Salud Pública 2012-2021: La Salud la construyes tú. Bogotá: Imprenta Nacional de Colombia; 2013.

8. Vega-Romero R, Acosta-Ramírez N. Mapeo y 
Análisis de los Modelos de Atención Primaria en Salud en los Países de América del Sur Mapeo de la APS en Colombia. Rio de Janeiro: Unión de Naciones Suramericanas-UNASUR. Instituto Suramericano de gobierno en Salud-ISAGS; 2014.

9. Giovanella L, De Almeida PF, Vega-Romero R, Oliveira S, Tejeira H. Panorama de la Atención Primaria de Salud en Suramérica: concepciones, componentes y desafíos. Saúde Debate. 2015; 39(105): 300-322. DOI: 10.1590/0103110420151050002002.

10. Organización Panamericana de la Salud, Ministerio de Salud y Protección Social, Instituto Nacional de Salud, Facultad Nacional de Salud Pública de la Universidad de Antioquia, Dirección de postgrados en Administración en Salud de la Pontificia Universidad Javeriana de Bogotá, Corporación de Secretarios de Salud Municipal - COSESAM. Documento marco Alianza por la APS para Colombia. Bogotá, 2014.

11. Ministerio de Salud y Protección Social. Política de Atención Integral en Salud: Un sistema de salud al servicio de la gente. Bogotá: Imprenta Nacional de Colombia; 2016.

12. Arboleda LM. El grupo de discusión como aproximación metodológica en investigaciones cualitativas. Rev Fac Nac Salud Pública. 2008; 26(1): 69-77.

13. Elo S, Kyngäs H. (2008). The qualitative content analysis process. J Advanc Nurs. 2008; 62: 107-111.

14. Ruiz-Rodríguez M, Acosta-Ramírez N, RodríguezVillamizar L, Uribe LM, León-Franco M. Experiencia de implementación de un modelo de Atención Primaria. Rev Salud Pública. 2011; 13(6): 885-896.

15. Chiavenato I. Administración de Recursos Humanos: El capital humano de las organizaciones. 9ed. México: McGraw-Hill, Interamericana Editores; 2011.

16. Rey-Gamero AG, Acosta-Ramírez N. El enfoque de competencias para los equipos de Atención Primaria en Salud. Una revisión de literatura. Rev Gerenc Pol Salud. 2013; 12(25): 28-39.

17. Molina G, Oquendo-Lozano T, Rodríguez-Garzón S, Montoya-Gómez N, Vesga-Gómez C, LagosCampos N, et al. Gestión del talento humano en salud pública. Un análisis en cinco ciudades colombianas, 2014. Rev Gerenc Pol Salud. 2016; 15(30): 108-125. DOI: http://dx.doi.org/10.11144/ Javeriana.rgyps 15-30.gtsp.

18. Vargas I, Unger JP, Mogollón-Pérez AS, Vázquez ML. Effects of managed care mechanisms on access to healthcare: results from a qualitative study in
Colombia. Int J Health Plann Manage. 2013; 28(1): e13-33. DOI: 10.1002/hpm.2129.

19. Molina G, Vargas J, Muñoz IF, Acosta JJ, Sarasti D, Higuita Y, et al. Dilemas en las decisiones en la atención en salud. Ética, derechos y deberes constitucionales frente a la rentabilidad financiera en el sistema de salud colombiano. Rev Gerenc Polit Salud. 2010; Sup. 9(18): 103-117.

20. Peters DH, Adam T, Alonge O, Agyepong IA, and Tran N. Implementation Research: What it is and how to do it. BMJ. 2013; 347: f6753. DOI: https:// doi.org/10.1136/bmj.f6753.

21. Ramírez-Acosta N, Ruiz-Pollard, Romero-Vega R, Labonté R. Comprehensive Primary health care in South America: contexts, achievements and policy implications. Cad Saúde Pública. 2011; 27(10): 1875-1890. DOI: http://dx.doi.org/10.1590/S0102$311 \times 2011001000002$. 